\title{
An Unambiguous Deterministic Compressed Acquisition Technique for BOC Signal
}

\author{
Xiaoheng Tan, Yan Zhang, Qiang Yan, Tingting Pan and Xiaonan Hu \\ College of communication engineering, Chongqing University, Chongqing, \\ 400044, China \\ 20085060@cqu.edu.cn
}

\begin{abstract}
The long PN code in a Binary Offset Carrier (BOC) modulated signal has a higher rate than the GPS C/A code, which makes the acquisition process in the new global navigation satellite system (GNSS) much more complex. To achieve a fast acquisition of the GNSS signal with a reduced number of correlators and low computational complexity, a two-stage deterministic compressed (DS) GNSS acquisition technique has been proposed. Nevertheless, the ambiguous threat of BOC signal isn't taken into account in the DS technique. To eliminate the ambiguity threat in the acquisition process of BOC $(\mathrm{m}$, n) signal, an unambiguous deterministic compressed acquisition is proposed in this paper. The main idea of the proposed technique is to introduce a simplified GRASS algorithm in the second stage measurement. Theoretical analysis and simulation results show that, at the expense of some performance degradation the proposed technique can completely remove the undesired positive side peaks of $B O C(m, n)$ signal, and reduce the chance of false peak acquisition compared with the DS technique.
\end{abstract}

Keywords: Binary offset carrier (BOC), global navigation satellite system (GNSS), unambiguous acquisition, deterministic compressed

\section{Introduction}

With the advent of the new global navigation satellite system, split-spectrum type modulated signals, such as binary offset carrier (BOC) signals, have been adopted in the next-generation GNSS to provide a better spectral separation between the existing GNSS systems such as modernized GPS, Galileo, GLONASS and COMPASS. A BOC signal is defined as a spreading pseudo random noise (PRN) code modulated by a square-wave subcarrier, and it's denoted as $\operatorname{BOC}\left(f_{s c}, f_{c}\right)$ or $\operatorname{BOC}(\mathrm{m}, \mathrm{n})$, where $f_{s c}=m \times 1.023 \mathrm{MHz}$ is the subcarrier frequency and $f_{c}=n \times 1.023 \mathrm{MHz}$ is the chip rate[1]. Since the square wave subcarrier can either be sine or cosine phased, the BOC signal is usually denoted as BOCs $(\mathrm{m}, \mathrm{n})$ and $\mathrm{BOCc}(\mathrm{m}, \mathrm{n})$. It has been revealed that BOC modulation can improve the code tracking performance and provide the advantages of multipath effect reduction and narrow band interference rejection [2]. However, the PRN code in BOC modulation signal has a much higher chip rate than the one in a GPS C/A signal, for which a receiver should have a higher search resolution, and the acquisition process has increased complexity [3]. Additionally, the existence of subcarrier in a BOC signal induces side peaks on both side of the center peak of the autocorrelation function (ACF), which may bring ambiguity threat in acquisition and tracking processes under multipath and interference environment.

Since the acquisition process for a BOC signal requires elaborate work, many methods have been proposed to solve this problem [4-10]. Serial search tests all code phases oneby-one, which makes a very long search process [4]. On the contrary Parallel-correlatorbased technique completes the acquisition by testing the phase of every PRN code in 
parallel, which needs multiple times in number of parallel correlators than the GPS C/A code [5]. The fast Fourier transform (FFT)-based technique uses a digital signal processor for fast computation, but it requires much more complex multiplications than the traditional method [6]. Folding technique and dual folding technique [8,9] provide another valid approach to accelerate the acquisition process by folding local signal and the incoming signal, and in this method the code phases to be searched will be decreased proportional to the folding number, but it suffers from SNR degradation with the increase of folding number. A two-stage deterministic compressed GNSS acquisition technique has been proposed to reach a good compromise between the correlator number and the acquisition time [10]. But the authors in [10] only discussed the performance of this method when used on GPS signal and BOC $(n, n)$ signal, and both the transform matrix and the measurement matrix are not suitable for BOC $(m, n)$ signal, besides, the ambiguity threat was not taken into account.

In this paper, we improve the deterministic compressed GNSS acquisition technique by introducing new transform matrix and measurement matrix, and adding an improved GRASS technique in the second stage. The proposed technique is suitable for all BOC signal and the ambiguity threat is suppressed at the same time. We first describe the proposed technique for different BOC modulations, and then the performances of the proposed technique are analyzed. Next a comparison of the proposed technique to other techniques is discussed. Lastly the conclusions are given.

\section{Description of the Proposed Method}

The incoming BOC $(\mathrm{m}, \mathrm{n})$ signal in a GNSS receiver can be expressed as:

$$
r(t)=\sqrt{2 P} D(t-\tau) C(t-\tau) s c(t-\tau) e^{j\left(2 \pi\left(f_{I F}+f_{D}\right) t+\varphi\right)}+n(t)
$$

where $P, \tau, f_{I F}, f_{D}$ and $\varphi$ are the power, code delay, IF carrier frequency, Doppler frequency, and carrier phase of the received signal, respectively. $D(t)$ and $n(t)$ represent the navigation data at the rate of $R_{b}$ bps and the incoming Gaussian noise with two-side power spectral density $N_{0} / 2$, respectively. $P(t)$ is the spreading sequence at code frequency $f_{c}=n \times 1.023 \mathrm{MHz}, s c(t)$ is the subcarrier, and its mathematical representation is represented as

$$
s c(t)= \begin{cases}\operatorname{sgn}\left(\sin \left(2 \pi f_{s c} t\right)\right), & \text { for } \sin \mathrm{BOC} \\ \operatorname{sgn}\left(\cos \left(2 \pi f_{s c} t\right)\right), & \text { for } \cos \mathrm{BOC}\end{cases}
$$

where $f_{s c}=m \times 1.023 \mathrm{MHz}$ is the subcarrier frequency. $\alpha=2 \mathrm{~m} / n$ is the modulation order which is a positive integer. In practice, GNSS signal search is a 2-Dimensinal hypothesis testing, which will search both code phase and the Doppler frequency of the received signal. During the acquisition process, the receiver multiplies the received signal by $e^{-j 2 \pi\left(f_{I F}+f_{d}+p \Delta f\right) t}$ to remove the carrier frequency, where $f_{d}$ is the minimum Doppler frequency estimated by the receiver, $\Delta f$ is the frequency search step size, and $p\left(p=0,1,2, \cdots, p_{\max }\right)$ is the Doppler frequency index.

To keep the better precision in the estimation of the delay and phase for the incoming BOC signal, we choose a sampling frequency $f_{s}=\alpha f_{c}$, and the sampled received signal can be expressed as $\mathbf{r}=\{r[0], r[1], \cdots, r[\alpha L-1]\}^{T}$, where $L=1023$ is the code length of the PN code. As the BOC signal has a very sparse expression in the autocorrelation output, we can get the transform matrix $\boldsymbol{\Psi}$ :

$$
\psi_{l, k}=B\left[\left\lceil(k-l) T_{s} f_{c}\right\rceil(\bmod L)\right]
$$


where $B(t)=C(t) s c(t), l, k \in\{0,1,2, \cdots, \alpha L-1\}$. When $f_{d}+p \Delta f$ is closed enough to $f_{D}$, we can turn the received BOC signal into its sparse domain by multiplying it by the transform matrix $\Psi$,

$$
\mathbf{R}=\boldsymbol{\Psi} \mathbf{r}
$$

where $\mathbf{R}$ is a $K$-sparse signal with $K=2 \alpha-1$ that represents all code phases in $1 / \alpha$ chip resolution. The amplitude of $\mathbf{R}$ can be thought as the ACF of BOC (m, n) signal. We use $\mathbf{R}_{B}$ to represent the normalized ACF of BOC signal without noise [2]:

$$
R_{B}(\tau)= \begin{cases}(-1)^{k+1}\left[\frac{\tau(2 \alpha-2 k-1)}{T_{c}}-\frac{2(\alpha-1) k-2 k^{2}+\alpha}{\alpha}\right], \frac{k T_{c}}{\alpha} \leq \tau<\frac{(k+1) T_{c}}{\alpha} \\ (-1)^{k+1}\left[\frac{\tau(2 k-1)}{T_{c}}+\frac{(\alpha-k)(2 k-1)-k}{\alpha}\right], & \frac{(k-\alpha) T_{c}}{\alpha} \leq \tau<\frac{(k-\alpha+1) T_{c}}{\alpha} \\ 0, & |\tau|>T\end{cases}
$$

where $k=\{0,1, \cdots, \alpha-1\}$. Fig.1 shows the normalized ACF of the $\mathrm{BOC}(2 \mathrm{n}, \mathrm{n})$ signal and $\mathrm{BOC}(\mathrm{n}, \mathrm{n})$ signal, it's obviously that the number of side peaks becomes larger as the calue of $\alpha$ increases

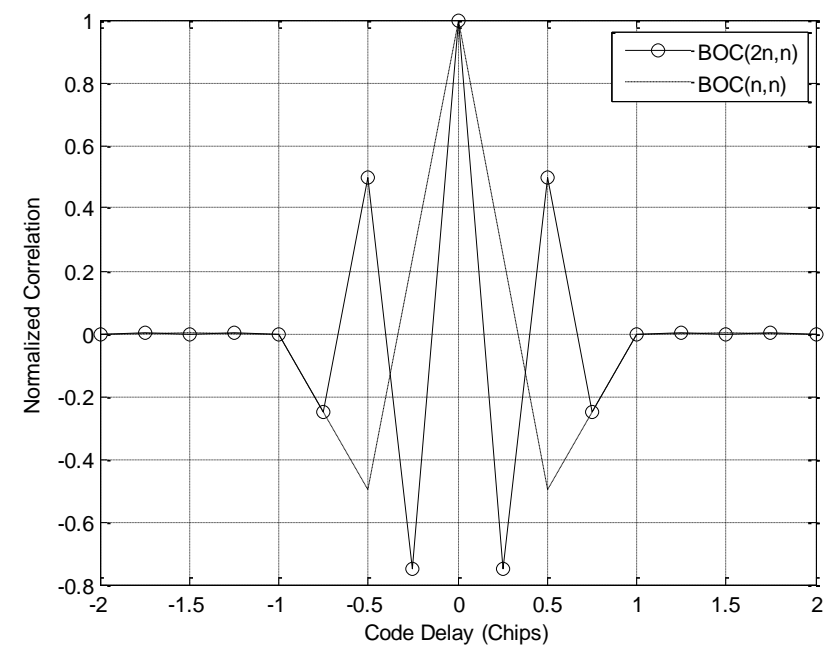

\section{Figure 1. The Envelop of $B O C(2 n, n)$ and $B O C(n, n)$ Signal Autocorrelation Functions}

In this paper, the proposed technique is divided into two stages.

\section{A. 1st-Stage}

The first stage measurement is the same as the one in [10]:

$$
\mathbf{y}_{1}=\boldsymbol{\Phi}_{1} \mathbf{R}=\boldsymbol{\Phi}_{1} \boldsymbol{\Psi r}
$$

where, the element of measurement matrix $\boldsymbol{\Phi}_{1}$ can be defined as

$$
\phi_{1_{m, l}}=\frac{(-1)^{l}}{\sqrt{M_{1}}} \mathbf{W}_{M_{01}}\left[m,\left\lceil\frac{l}{\eta}\right\rceil\right]
$$

where $1 \leq m \leq M_{1}, 1 \leq l \leq \alpha L, \mathbf{W}_{M_{01}}$ is a Walsh-Hadamard matrix of size $\left[M_{1} \times M_{1}\right]$, $M_{1}=2^{M_{01}}, \eta$ is called compression ratio, which satisfies $\eta M_{1} \square \alpha L$.

The first stage measurement can be implemented by the following steps: R:

Step1. Multiplying the sampled received signal $\mathbf{r}$ by the transform matrix $\boldsymbol{\Psi}$ to get 


$$
\mathbf{R}=\boldsymbol{\Psi} \mathbf{r} .
$$

Step2. Multiplying $\mathbf{R}$ by the measurement matrix $\boldsymbol{\Phi}_{1}$ to get the observed signal $\mathbf{y}_{1}$ :

$$
\mathbf{y}_{1}=\Phi_{1} \mathbf{R}=\Phi_{1} \Psi \mathbf{r}
$$

Step3. Calculating the FWHT transform of $\mathbf{y}_{1}$ :

$$
\mathbf{Z}_{1}=\left|\mathbf{z}_{1}\right|^{2}=\mid M_{1} \text { - point FWHT of }\left.\mathbf{y}_{1}\right|^{2} \text {. }
$$

Step4. Selecting maximum $N_{p}$ largest elements of $\mathbf{Z}_{1}$, which must be larger than the first-stage detection threshold $\gamma_{1}$ at the same time, and let $\mathbf{w}_{1}$ denote the index of the selected element:

$$
\mathbf{w}_{1}=\arg \left\{\left\{N_{p} \text { larg est elements of } \mathbf{Z}_{1}\right\} \cap\left\{\mathbf{Z}_{1} \geq \gamma_{1}\right\}\right\} .
$$

Step5. If $\left\|\mathbf{w}_{1}\right\|_{0}>0$, proceed to the 2nd-stage with $\mathbf{W}_{1}$ :

$$
\mathbf{W}_{1}=\bigcup_{w_{1} \in \mathbf{w}_{1}}\left\{\eta w_{1}+1, \eta w_{1}+2, \cdots, \min \left\{\eta\left(w_{1}+1\right)+1, \alpha L\right\}\right\},
$$

Otherwise, repeat 1st-stage with $p=p+1$.

During the first stage measurement, $z_{1} \in \mathbf{z}_{1}$ can be expressed as:

$$
z_{1}(m)=\sum_{n=\eta(m-1)+1}^{K} R(n)
$$

where $0 \leq m \leq M_{1}, K=\min \{\eta m, \alpha L\}$, thus it can be considered that the first stage measurement completes the signal compression by adding every $\eta$ elements of $\mathbf{r}$. The code phases to be searched are directly decreased proportional to $\eta$.

\section{A. 2nd-Stage}

According to the previous introduction, the ACF of BOC signal has multi peaks which may bring an ambiguity threat in signal acquisition and tracking. To get rid of this problem, we apply the improved GRASS technique in the second stage measurement of the proposed technique.

BOC signal can be considered as a special case of step-shape code symbol (SCS) signals whose shape vector is $\mathbf{d}_{B}=[1,-1, \cdots, 1,-1]_{\alpha \times 1}^{\mathrm{T}}$. Thus we can get any BOC signal by multiplying each chip of the PN code by corresponding $\mathbf{d}_{B}$. In this stage, a new synthetic ACF $\mathbf{R}^{\prime}$, is applied to accomplish the acquisition process:

$$
\mathbf{R}_{\mathrm{B}}^{\prime}=\mathbf{R}_{\mathrm{B}}^{2}-\beta \mathbf{R}_{B / L}^{2}
$$

where, $\beta=2 \alpha-3, \mathbf{R}_{B / L}=\boldsymbol{\Psi}_{L} \mathbf{r}, \psi_{L_{l, k}}=L\left[\left\lceil(k-l) T_{s} f_{c}\right\rceil(\bmod L)\right]$ and $L(t)$ is the local SCS signal whose shape vector $\mathbf{d}_{L}=\left[d_{0}, d_{1}, \cdots, d_{\alpha-1}\right]_{\alpha \times 1}^{\mathrm{T}}$,

$$
\left\{\begin{array}{l}
d_{0}=d_{\alpha-1}=\frac{\alpha-1}{\sqrt{2 \alpha-3}} \\
d_{i}=d_{\alpha-i-1}=\frac{(-1)^{i-1}}{\sqrt{2 \alpha-3}},\left(i=1,2, \cdots, \frac{\alpha}{2}-1\right)
\end{array}\right.
$$

According to the description above, we can get $\boldsymbol{\Psi}_{L}$ easily by doing some calculations of $\boldsymbol{\Psi}: \psi_{L_{l, k}}=\psi_{l, k} d_{k}$, where $d_{k} \in \mathbf{d}=\left[\mathbf{d}_{B}^{\mathrm{T}} \bullet \mathbf{d}_{L}^{\mathrm{T}} \mathbf{d}_{B}^{\mathrm{T}} \bullet \mathbf{d}_{L}^{\mathrm{T}} \cdots \mathbf{d}_{B}^{\mathrm{T}} \bullet \mathbf{d}_{L}^{\mathrm{T}}\right]_{1 \times \alpha L}^{\mathrm{T}}$. The second stage measurement of the proposed method can be implemented by the following steps:

Step1. Let $M_{02}$ equal $\left\lceil\log _{2}\left\|\mathbf{W}_{1}\right\|_{0}\right\rceil, v$ be the index of elements of $\mathbf{W}_{1}$, and generate another Walsh-Hadamard matrix $\mathbf{W}_{M_{02}}$ with the size of $\left[M_{2} \times M_{2}\right]$, where $M_{2}=2^{M_{02}}$. 
Step2. Generate $\mathbf{R}_{2}$ and the measurement matrix $\boldsymbol{\Phi}_{2}$ :

$$
R_{m, v}^{\prime}=\mathbf{R}^{2}(l)-\beta \mathbf{R}_{B / L}^{2}, \mathbf{\Phi}_{2}=\mathbf{W}_{M_{02}} / \sqrt{M_{2}},
$$

where $v=\left\{1,2 \cdots,\left\|\mathbf{W}_{1}\right\|_{0}\right\}, \quad v=\left\{1,2 \cdots,\left\|\mathbf{W}_{1}\right\|_{0}\right\}, \quad R_{2_{m, v}}$ is the element of $\mathbf{R}_{2}$, $\mathbf{R}_{2} \in \square^{M_{2} \times M_{2}}, R(l), R_{B / L}(l)$ is the $l$ th element of $\mathbf{R}$ and $\mathbf{R}_{B / L}$.

Step3. Get the observed signal $\mathbf{y}_{2}$ :

$$
\mathbf{y}_{2}=\boldsymbol{\Phi}_{2} \mathbf{R}^{\prime}
$$

Step4. Calculating the FWHT transform of $\mathbf{y}_{2}$ :

$$
\mathbf{Z}_{2}=\left\{M_{2}-\text { point FWHT of } \mathbf{y}_{2}\right\} \text {. }
$$

Step5. Finding the index of the largest element in $\mathbf{Z}_{2}$, which is larger than the secondstage detection threshold $\gamma_{2}$ :

$$
\mathbf{w}_{2}=\arg \left\{\left\{\text { larg est element of } \mathbf{Z}_{2}\right\} \cap\left\{\mathbf{Z}_{2} \geq \gamma_{2}\right\}\right\} .
$$

Step6. If $\left\|\mathbf{w}_{2}\right\|_{0}>0$, return $\left\|\mathbf{w}_{2}\right\|_{0}>0$, and the acquisition process is accomplished, otherwise, repeat 1st-stage with $p=p+1$.

The method used in the second stage of the proposed technique is not the same as the GRASS technique. After the first stage measurement, the phase search range has been reduced from $\alpha L$ to $\left\|\mathbf{W}_{1}\right\|_{0}$, thus we just need calculate $\left\|\mathbf{W}_{1}\right\|_{0}$ elements of $\mathbf{R}^{\prime}$ and $\boldsymbol{\Psi}_{L}$ here instead of $\alpha L$ elements in GRASS. Compared with the GRASS, the proposed technique needs much less computation and hard resources.

\section{Performance Analysis}

To analyze the performance of the proposed technique, in this section, we calculate the detection and false alarm probabilities. Here we assume $N_{p}=1$ as the simplest implementation of the proposed technique, and the incoming noise is Gaussian noise. For the first stage measurement, when there is no signal present, the distribution of $Z_{1} \in \mathbf{Z}_{1}$ is a central chi-square distribution with two degrees of freedom, the probability distribution function of $Z_{1}$ can be expressed as:

$$
p_{f a 1}\left(Z_{1}\right)=\frac{1}{2 \sigma_{1}^{2}} \exp \left(-\frac{Z_{1}}{2 \sigma_{1}^{2}}\right)
$$

where $\sigma_{1}^{2}=(2 \eta-1) \alpha L N_{0} / 2$. We can get the false alarm probability of each test cell according to the definition of false alarm probability.

$$
P_{c e l l, f a 1}=\int_{\gamma_{1}}^{\infty} p_{f a 1}\left(Z_{1}\right) \mathrm{d} Z_{1}=\exp \left(-\frac{\gamma_{1}}{2 \sigma_{1}}\right)
$$

The overall false alarm probability is:

$$
P_{f a 1}=1-\left[1-P_{\text {cell, fa1 }}\right]^{M_{1}} \approx M_{1} P_{\text {cell }, f a 1}
$$

For signal present case, the distribution of $Z_{1} \in \mathbf{Z}_{1}$ is a noncentral chi-square distribution with two degrees of freedom, the PDF of $Z_{1}$ can be expressed as:

$$
p_{d 1}\left(Z_{1}\right)=\frac{1}{2} \exp \left(-\frac{\lambda_{1}}{2}\right) \exp \left(-\frac{Z_{1}}{2 \sigma_{1}^{2}}\right) \sum_{m=0}^{\infty} \frac{\left(\frac{\alpha}{\sigma_{1}^{2}}\right)^{m} \lambda^{m}}{\Gamma\left(\frac{1}{2}+m\right) 2^{2 m} m !}
$$


where $\lambda=S_{1}^{2} / \sigma_{1}^{2}$. The signal amplitude $S_{1}$ in the first stage measurement can be expressed as:

$$
S_{1}= \begin{cases}\alpha \sqrt{2 P} L \sum_{i=0}^{\eta-1} R_{B}\left(\left(i+1-\left(l_{T}(\bmod \eta)\right)\right) \frac{T_{c}}{\alpha}\right), & l_{T}(\bmod \eta) \neq 0 \\ \alpha \sqrt{2 P} L \sum_{i=0}^{\eta-1} R_{B}\left(\frac{i T_{c}}{\alpha}\right), & l_{T}(\bmod \eta)=0\end{cases}
$$

where $l_{T}\left(1 \leq l_{T} \leq \alpha L\right)$ is the index of the maximum peak in $\mathbf{R}$. For BOC(n, n) signal when $\eta>3, \quad \bar{S}_{1}=\left(2-\frac{1}{\eta}\right) 2 \sqrt{2 P} L ;$ for $\operatorname{BOC}(2 \mathrm{n}, \mathrm{n})$ signal when $\eta>7$, $\bar{S}_{1}=\left(4-\frac{4}{\eta}\right) 4 \sqrt{2 P} L$.

The detection probability is defined as:

$$
P_{D}=\int_{V_{T}}^{\infty} p_{d}(x) \mathrm{d} x
$$

Then we can establish the detection probability of the proposed technique by applying (14) in (16), the detection probability of the first measurement can be expressed as [10]:

$$
\begin{aligned}
P_{D 1} & \geq \sum_{n=0}^{M_{1}-1} \frac{(-1)^{n}}{n+1}\left(\begin{array}{c}
M_{1}-1 \\
n
\end{array}\right) \exp \left(-\frac{n S_{1}^{2}}{2(n+1) \sigma_{1}^{2}}\right) \\
& \times Q\left(\sqrt{\frac{2 S_{1}^{2}}{2(n+1) \sigma_{1}^{2}}}, \sqrt{\frac{2(n+1) \gamma_{1}}{2 \sigma_{1}^{2}}}\right)
\end{aligned}
$$

For the second stage measurement, we use the improved GRASS technique to eliminate the ambiguity threat. When there is no signal present, both of the first term and the second term in (9) follow central chi-square distribution with two degrees of freedom, but with different variances $\sigma_{2,1}^{2}=\alpha L N_{0} / 2, \sigma_{2,2}^{2}=\beta \sigma_{2,1}^{2}=\beta \alpha L N_{0} / 2$. Then we can give the false alarm probability of each test point in the second term expression by exploiting the derivations in [2]:

$$
P_{c e l l, f a 2}=\frac{1}{1+\beta} \exp \left(-\frac{\gamma_{2}}{\alpha L N_{0}}\right)
$$

The overall false alarm probability is:

$$
P_{f a 2}=1-\left[1-P_{\text {cell }, f a 2}\right]^{M_{2}} \approx M_{2} P_{\text {cell }, f a 2}
$$

For signal present case, when the code delay is small, the first term in (9) follows noncentral chi-square distribution with two degrees of freedom, in which the noncentrality parameter is $\lambda_{2,1}=S_{2,1}^{2} / \sigma_{2,1}^{2}, S_{2,1}=\alpha \sqrt{2 P} L$. The second term still follows central chi-square distribution with two degrees of freedom, with $\sigma_{2,2}^{2}=\beta \sigma_{2,1}^{2}=\beta \alpha L N_{0} / 2$. Then the detection probability of the second stage is [2]:

$$
\begin{aligned}
P_{D 2} & =\int_{\gamma_{2}}^{+\infty} \frac{1}{2(1+\beta) \sigma_{2,1}^{2}} \exp \left(-\frac{Z_{2}}{2 \beta \sigma_{2,1}^{2}}\right) \exp \left(-\frac{S_{2,1}^{2}}{2(1+\beta) \sigma_{2,1}^{2}}\right) \\
& \times Q\left(\frac{S_{2,1}^{2}}{\sigma_{2,1}^{2}} \sqrt{\frac{\beta}{(1+\beta)}}, \sqrt{\frac{2(1+\beta) Z_{2}}{\beta \sigma_{2,1}^{2}}}\right) d Z_{2}
\end{aligned}
$$




\section{Results and Discussions}

In this section, the proposed technique is tested for a GNSS receiver in an acquisition process of $\mathrm{BOC}(10,5)$ signal, sampling frequency $f_{s}=4 f_{c}$, correlation length is $4 L=4096$. Figure 2 shows the detection probability for $\operatorname{BOC}(10,5)$ versus SNR with a fixed false alarm probability $P_{f a}=10^{-5}$ for $M_{1}=\{128,256,512\} \quad$ and $N_{p}=\left\{1,\left\lceil 0.05 M_{1}\right\rceil,\left\lceil 0.1 M_{1}\right\rceil\right\}$.

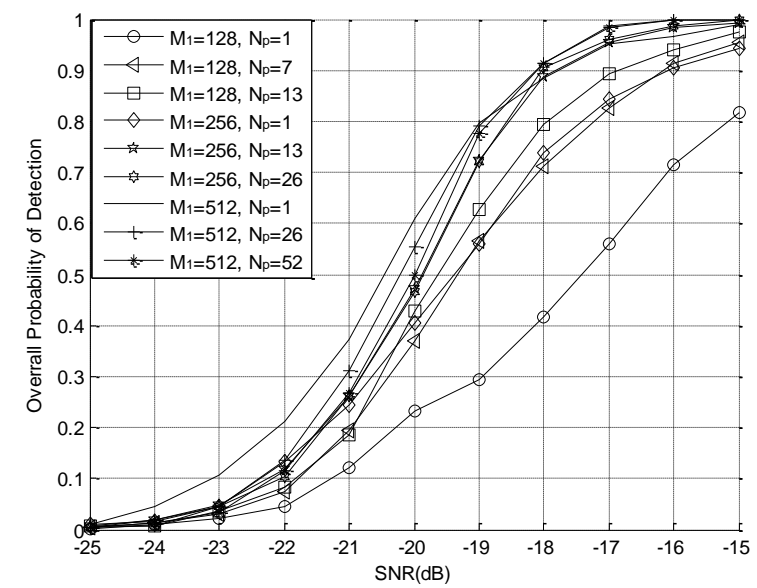

Figure 2. Overall Detection Probability of the Proposed Technique with versus SNR, $M_{1}$ and $N_{p}$

The detection probability is obtained from the $10^{4}$ Monte Carlo simulation in the AWGN channel. It can be observed from Figure 2 that, for a fixed $N_{p}$, as the value of $M_{1}$ increases, the performance of the proposed technique gets better, because a greater compression ratio may bring a larger loss. Besides, when the value of $M_{1}$ is small, a greater $N_{p}$ brings a better detection performance, but as $M_{1}$ increases, this conclusion do not hold water certainly. This is because for the fixed false alarm probability and $M_{1}$, the threshold of the second stage measurement increases with the increase of $N_{p}$, when the value of $M_{1}$ is high, the influence of $N_{p}$ on the threshold is not negligible.

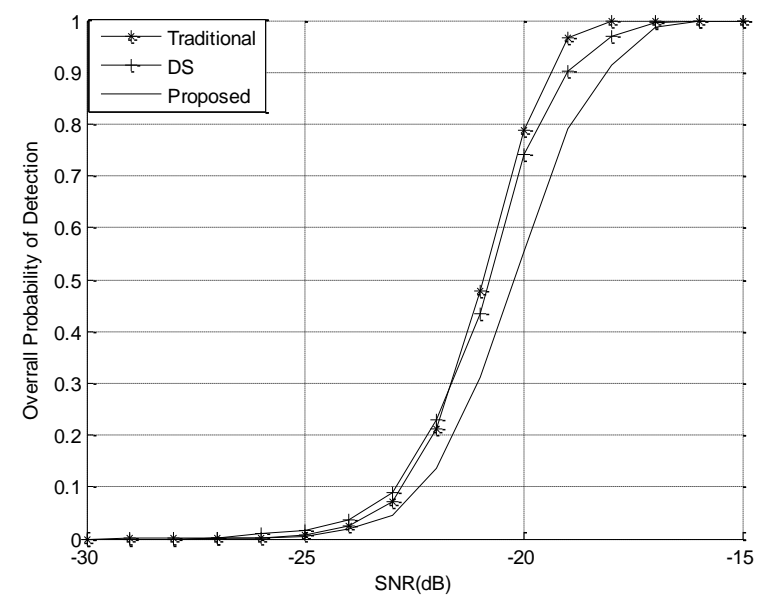

Figure 3. Overall Detection Probability of the Proposed Technique, the DS Technique and Traditional Technique 
In [10], the deterministic compressed GNSS acquisition technique has almost the same acquisition performance as the traditional technique with small $\eta$ and $N_{p}\left(1 \square N_{p} \square M_{1}\right)$, a comparison among the proposed technique, the deterministic compressed acquisition technique and the traditional acquisition technique is shown in Figure 3. It can be seen that, when $\eta$ is small and $1 \square N_{p} \square M_{1}$, the sensitivity of the proposed technique degrades about $2 \mathrm{~dB}$ compared with the traditional acquisition technique. This is because the sum operation in (9) introduces more noise. But the same as the DS technique, the proposed technique needs $M_{1}+\eta M_{2}$ correlators, which is much less than the conventional parallel-correlator-based technique.

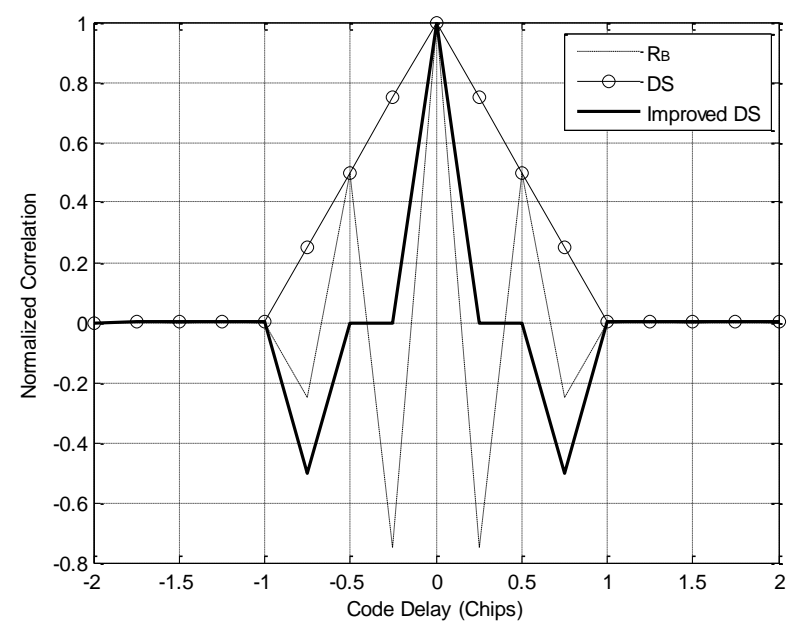

\section{Figure 4. The ACF of BOC $(10,5)$ Signal, the Second Stage Measurement Test Criterion of the DS Technique and the Proposed Technique}

Figure 4 shows the $\mathrm{ACF} R_{B}$ of $\mathrm{BOC}(10,5)$, the second stage measurement test criterion $Z_{2, D S}$ of the deterministic compressed acquisition technique in [10] and the second stage measurement test criterion $Z_{2 \text {,Proposed }}$ of the proposed technique. All of the three curves are normalized. On the face of it, the test criterion curve of DS technique in [10] is triangular and has only one peak, but the ambiguous threat still exists, for $Z_{2, D S}$ can be considered as the absolute value of $R_{B}$. However for the proposed technique, the test criterion curve has only one positive peak, which is much narrower than the one in DS technique. This makes the proposed technique more reliable than the DS technique. In [2], the test criterion curve of GRASS also has one positive peak and the peak is narrower than the main peak of ACF of BOC $(10,5)$, but Fig. 4 shows the opposite result, this is because the sampling frequency in this paper is lower than [2], which brings the difference.

\section{Conclusion}

In this paper, a two-stage measurement technique has been proposed to accomplish the unambiguous acquisition of $\mathrm{BOC}(\mathrm{m}, \mathrm{n})$ signal. The detection performance and the test criterion in the second measurement of the proposed the technique have been analyzed and compared with the DS technique. It has been shown that, at the cost of some degradation of detection probability in acquisition the proposed technique can eliminate the ambiguity threat in the acquisition process by removing the undesired positive side peaks, which is much more reliable than the DS technique. It is therefore more reliable than the DS technique. 


\section{Acknowledgements}

This work is supported by the open research fund of Chongqing Key Laboratory of Emergency Communications, and the Fundamental Research Funds for the Central Universities (106112013CDJZR165501)

\section{References}

[1] J. W. Betz, "Binary offset carrier modulations for radionavigation," J. Inst.Navigation, vol. 48, no. 4, (2001) May, pp. 227-246.

[2] Z. Yao, "Unambiguous Sine-Phased Binary Offset Carrier Modulated Signal Acquisition Technique," IEEE Trans. Wireless Commun., vol. 9, no. 2, (2010) February, pp. 577-580.

[3] O. Julien, C. Macabiau,M. E. Cannon and G. Lachapelle, "ASPeCT: Unambiguous Sine-BOC(n, n) Acquisition/Tracking Technique for Navigation Applications," IEEE Trans. on Aero. Electr. Sys., vol. 43, no. 1, (2007) January, pp.150-162.

[4] A. Polydoros and G. L. Weber, "A unified approach to serial search spread-spectrum code acquisitionpart I and II,” IEEE Trans. Commun., vol. 32, no. 5, (1984) May, pp. 542-560.

[5] U. Cheng, W. J. Hurd, and J. I. Statman, "Spread Spectrum Code Acquisition in The Presence of Doppler Shift and Data Modulation," IEEE Trans. Commun., vol. 38, no. 2, (1990) February, pp. 241250.

[6] D. Akopian, "Fast FFT based GPS satellite acquisition methods," IEEE Proc.-Radar Sonar Navig., vol. 152, no. 4, (2005) August.

[7] H. Li, M. Lu, X. Cui and Z. Feng, "Generalized Zero-Padding Scheme for Direct GPS P-code Acquisition,” IEE Trans. Wirel. Commun., vol. 6, no. 10, (2009) June, pp. 2866-2871.

[8] C. Yang, J. Vasquez, and J. Chaffee, "Fast direct P(Y)-code acquisition using XFAST," Proc. Of ION GPS-99, (1999) September, pp. 317-324.

[9] H. Li, X. Cui, M. Lu, and Z. Feng, "Dual-folding-based rapid search method for long PN-code acquisition,” IEEE Trans. Wireless Commun., vol. 7, no. 12, (2008) December, pp. 5286-8296.

[10] S.-H. Kong, "A Deterministic Compressed GNSS Acquisition Technique," IEEE Trans. Vehic. Techn., vol. 62, no. 2, (2013) February, pp. 511-521.

[11] Y. Zhou, X Hu, T. Ke, and Z. Tang, "Ambiguity Mitigating Technique for Cosine-Phased Binary Offset Carrier Signal,” IEEE Trans. Wireless Commun., vol. 11, no. 6, (2012) June, pp. 1981-1984.

[12] C. Yu, J. In Park, Y. Lee, and S. Yoon, "Unambiguous BOC signal acquisition based on recommendation of sub-correlations," in Proc. 8th Adv. Int. Conf. Telecommun., (2012), pp. 56-61.

[13] F. Benedetto, G. Giunta, E. S. Lohan, "Analysis of Side Loes Cancellation Methods for BOCcos(n,m) Signal [J]", IEEE Transactions on Vehicular Technology, vol. 62, no. 3, (2013), pp. 1350-1355.

[14] C. Yu, J. In Park, Y. Lee, and S. Yoon, "Unambiguous BOC signal acquisition based on recommendation of sub-correlations," in Proc. 8th Adv. Int. Conf. Telecommun., (2012), pp. 56-61.

\section{Authors}

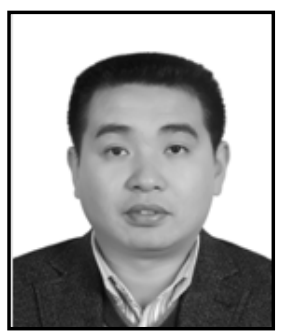

Xiaoheng Tan, received the B.S. degree in Chongqing University, Chongqing, China, in 1998, and received his $\mathrm{PhD}$. Degrees in Chongqing University, in 2003. He went to the University of Queensland as a visiting scholar, during June 2008 to May 2009. He is now a professor and doctoral tutor at the college of communication engineering of Chongqing University, Chongqing University, Chongqing, China. His research interests include modern communications technologies and systems (CDMA spread spectrum technology and communication, the next generation broadband wireless mobile communication technology, satellite communications, etc.), communications signal processing. 


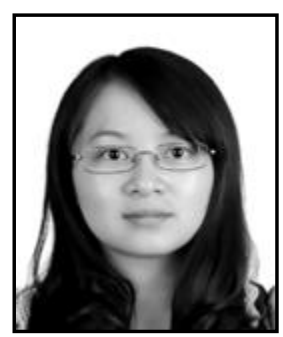

Yan Zhang, received the B.S. degree in Electronic information Engineering from Chongqing University, Chongqing, China, in 2012. She is working towards the M.S. degree in communication and information system at Chongqing University, Her current interests include wireless communication, satellite positioning and navigation. 